\title{
Electromagnetic interferometry in wavenumber and space domains in a layered earth
}

\author{
Jürg Hunziker ${ }^{1}$, Evert Slob ${ }^{1}$, Yuanzhong Fan², Roel Snieder ${ }^{3}$, and Kees Wapenaar ${ }^{1}$
}

\begin{abstract}
With interferometry applied to controlled-source electromagnetic data, the direct field and the airwave and all other effects related to the air-water interface can be suppressed in a data-driven way. Interferometry allows for retreival of the scattered field Green's function of the subsurface or, in other words, the subsurface reflection response. This reflection response can then be further used to invert for the subsurface conductivity distribution. To perform interferometry in 3D, measurements on an areal grid are necessary. We discuss 3D interferometry by multidimensional deconvolution in the frequency-wavenumber and in the frequency-space domains and provide examples for a layered earth model. We use the synthetic aperture source concept to damp the signal at high wavenumbers to allow large receiver sampling distances. Interferometry indeed increases the detectability of a subsurface reservoir. Finally, we discuss the dependency of the accuracy of the retrieved reflection response on the two crucial parameters: the conductivity of the seabed at the receiver location and the stabilization parameter of the least-squares inversion.
\end{abstract}

\section{INTRODUCTION}

A typical marine controlled-source electromagnetics (CSEM) survey consists of multicomponent ocean-bottom receiver stations and an electric source, which is towed by a boat over the receiver stations. A detailed overview of the method is given by Constable and Srnka (2007). The low-frequency signal diffuses from the source not only through the subsurface to the receivers, but also via the sea surface and directly through the water-layer. The so-called "airwave" is a signal that travels vertically upward from the source, horizontally in the air along the sea surface and, eventually, vertically down to the receivers at the ocean bottom. This is the direct airwave and the part of the direct airwave that diffuses down into the subsurface and reflects off conductivity contrasts can also contribute significantly to the measured response (see several monitoring studies, e.g., Wirianto et al., 2011). The airwave is only attenuated while traveling through the water, but not while propagating through the air. Consequently, its contribution is maximal in shallow water situations because of the short travel path that attenuates the airwave, and at receiver stations at large horizontal distances from the source because signals traveling along other paths have attenuated more until they are recorded at those receivers. In this way, the direct airwave is covering a possible signal from a subsurface reservoir. In contrast, the direct field contributes to the recorded field mainly at near offset receivers (Amundsen et al., 2006). Consequently, subsurface reflections can only be detected and interpreted at intermediate offsets.

In a sophisticated processing and interpretation scheme, CSEM data, i.e., the electromagnetic fields in the frequency-space domain as a function of offset from the source, are inverted for the subsurface conductivity distribution (for an overview see Avdeev, 2005). Due to the low-frequency signal, CSEM alone is not able to produce a high-resolution image of the subsurface. Therefore, structural information from seismics is often included in an inversion scheme (MacGregor and Sinha, 2000; Harris et al., 2009; Key, 2009).

Interferometry is mainly investigated in seismics (for an overview see Schuster, 2009; Wapenaar et al., 2010a, 2010b), but can also be applied to electromagnetic wave fields and diffusive electromagnetic fields. The main purposes of applying interferometry by multidimensional deconvolution (MDD) to marine CSEM data are to suppress the airwave in a data-driven way and to minimize source position and orientation uncertainty. Interferometry by MDD, first, replaces the overburden, i.e., the medium above the receivers, with a

Manuscript received by the Editor 16 December 2011; revised manuscript received 11 January 2013; published online 29 April 2013.

${ }^{1}$ Delft University of Technology, Section of Applied Geophysics and Petrophysics, Department of Geotechnology, Delft, The Netherlands. E-mail: j.w .hunziker@tudelft.nl; e.c.slob@tudelft.nl; c.p.a.wapenaar@tudelft.nl.

${ }^{2}$ Shell International Exploration and Production, Houston, Texas, USA. E-mail: yuanzhong.fan@shell.com.

${ }^{3}$ Colorado School of Mines, Center for Wave Phenomena, Golden, Colorado, USA. E-mail: rsnieder@mines.edu.

(C) 2013 Society of Exploration Geophysicists. All rights reserved. 
homogeneous half-space; second, it suppresses the direct field and, third, it redatums the source to a receiver position. In other words, the scattered field Green's function of the subsurface, henceforth called "subsurface reflection response," is retrieved, which is ideally free of any interactions between the air and the water-layer and, therefore, also free of the airwave. Uncertainty related to the source position and orientation is minimized because the source is redatumed in a data-driven way to a receiver whose location is normally better known than the location of the source. We expect that an inversion scheme, which uses the reflection response as input data, features a better-defined solution space because the objective function to be minimized does not contain strong events such as the airwave or the direct field, nor large uncertainties related to the source position and orientation, but ideally depends only on the subsurface response.

A derivation of the method starting with a reciprocity theorem of the convolution type for one-way fields and a 2D numerical example of electromagnetic interferometry are given in Wapenaar et al. (2008). A comparable reciprocity theorem, but of the correlation type, is in electromagnetics known as the reciprocity theorem of Lorentz (1896). Similar to electromagnetic interferometry is the method described by Nordskag et al. (2009). Our approach consists roughly of two steps: First, the multicomponent electromagnetic fields are decomposed into downward and upward decaying components. In the second step, the reflection response is retrieved by MDD. For the decomposition step, it is assumed that the data are properly sampled and that the receiver positions and orientations are known. The implementation used in this paper requires that the receivers are located in a plane of laterally invariant material parameters. Only the material parameters at the receiver level need to be known. No information about the water-layer or the source position and orientation are required. Because the receivers are located at the ocean bottom and because the horizontal components of the electromagnetic field are continuous across the interface, one can choose to use the material parameters of the seabed or those of the water for the decomposition. Eventually, this choice determines the material parameters of the half-space, which replaces the overburden. Although van den Berg et al. (2008) chose the material parameters of the water-layer for the decomposition, we follow Wapenaar et al. (2008) and use the material parameters of the seabed.

As already mentioned, for data-driven methods to work, the electromagnetic fields need to be properly sampled. This means that they need to be sampled sufficiently dense, for a large enough offset range and without gaps. Hunziker et al. (2012b) have shown that the largest possible receiver sampling distance depends on the height of the source above the receivers and the length of the source antenna. The receiver sampling distance is required to be smaller than the larger of these two parameters. Because this leads to impractically dense receiver spacings, Hunziker et al. (2012b) propose to exploit the oversampling on the source side by creating synthetic aperture sources introduced by Fan et al. $(2010,2011)$ to overcome these sampling restrictions. They show, on 2D synthetic data sets which are contaminated with noise and with receiver position and orientation errors, the applicability of synthetic aperture sources in combination with interferometry by MDD. Synthetic aperture sources damp the high-wavenumber content, which is mainly caused by the direct field and near surface reflections, without affecting a signal from a deeper reservoir.
Because CSEM is a low-frequency technique with an accordingly large wavelength, the recordings are always in the near-field, which requires a processing that takes the complete field into account. The 2D-processing developed by Hunziker et al. (2012b) can not be directly applied to $3 \mathrm{D}$ data because information about the second horizontal direction is missing. In this paper, we extend the 2D processing scheme developed by Hunziker et al. (2012b) to 3D data. There are two options to apply interferometry by MDD to 3D data: The first option is synthesized-2D interferometry, which only uses one receiver line as in the $2 \mathrm{D}$ case but, additionally, source lines parallel to the line of receivers. These source lines are used to synthesize an infinitely long source in the crossline direction to allow for $2 \mathrm{D}$ processing. This approach is discussed by Hunziker et al. (2012a). The second option is 3D interferometry, which requires recordings on a grid. This paper focuses on the second option. Algorithms for 3D interferometry in the wavenumber and in the space domain are presented. Subsequently, we show that the reservoir detectability has increased by applying interferometry and we investigate the impact of the choice of the conductivity at the receiver level and the choice of the stabilization parameter in the leastsquares inversion on the retrieved reflection response.

\section{THEORY}

The method consists of three major steps, which are described briefly in the following paragraphs. These steps are: (1) A synthetic aperture source is created to damp the signal at large wavenumbers, thereby reducing the effect of aliasing caused by the sparsely sampled data on the receiver side, which is common for CSEM data (Fan et al., 2010, 2011). (2) The multicomponent electromagnetic fields are decomposed into upward and downward decaying components (Amundsen et al., 2006; Slob, 2009). (3) The reflection response is retrieved by an MDD (Wapenaar et al., 2008; Nordskag et al., 2009).

\section{Synthetic aperture source}

To create a synthetic aperture source, recordings from different source positions are weighted and subsequently summed. Note that this does not include any steering of the source. We use the following Gaussian distribution function $f(x, y)$ as weighting function for the source positions

$$
f(x, y)=\exp \left(-\frac{\left(x-x_{\text {syn }}\right)^{2}+\left(y-y_{\text {syn }}\right)^{2}}{2 l^{2} / \nu^{2}}\right),
$$

where $x$ and $y$ represent the position of the source in inline and crossline directions, respectively, and, accordingly, $x_{\text {syn }}$ and $y_{\text {syn }}$ give the center of the synthetic aperture source. The parameter $l$ is the length of the source array in meters and $\nu$ is a parameter chosen empirically.

\section{Decomposition}

The transverse electromagnetic field components are Fourier transformed to the frequency-wavenumber domain. The 2D Fourier transform of a function $\hat{u}\left(x, y, z_{1}, \omega\right)$ in the frequency-space domain is defined as $\tilde{u}\left(k_{x}, k_{y}, z_{1}, \omega\right)=\iint_{-\infty}^{\infty} \exp \left(j k_{x} x+j k_{y} y\right) \hat{u}(x, y$, $\left.z_{1}, \omega\right) \mathrm{d} x \mathrm{dy}$. The circumflex denotes the frequency-space domain and the tilde the frequency-wavenumber domain, $k_{x}$ and $k_{y}$ are the inline and crossline wavenumber, respectively, $z_{1}$ is the receiver 
depth (in our case at the ocean bottom) and $\omega$ is the angular frequency. The inverse Fourier transform is given by $\hat{u}(x, y$, $\left.z_{1}, \omega\right)=1 /\left(4 \pi^{2}\right) \iint_{-\infty}^{\infty} \exp \left(-j k_{x} x-j k_{y} y\right) \tilde{u}\left(k_{x}, k_{y}, z_{1}, \omega\right) \mathrm{d} k_{x} \mathrm{~d} k_{y} . \quad$ A matrix multiplication of the electromagnetic field with the decomposition matrix $\tilde{\mathbf{L}}^{-1}$ given by Slob (2009) decomposes the field into upward and downward decaying transverse magnetic (TM) and transverse electric (TE) field potentials at each location in the horizontal wavenumber-domain, according to

$$
\tilde{\mathbf{P}}=\tilde{\mathbf{L}}^{-1} \tilde{\mathbf{Q}},
$$

where $\tilde{\mathbf{P}}=\left(\tilde{P}^{\mathrm{TM},+}, \tilde{P}^{\mathrm{TE},+}, \tilde{P}^{\mathrm{TM},-}, \tilde{P}^{\mathrm{TE},-}\right)^{T}$. The superscript $T$ means matrix transposition and the superscripts TE and TM indicate the type of mode, whereas the plus-sign indicates the downward diffusing mode and the minus-sign indicates the upward diffusing mode. Note that we follow here the standard definition of TE- and TM-modes, which says that for a vertically chosen reference axis, the vertical component of the electric field is zero for the TE-mode and the vertical component of the magnetic field is zero for the TM-mode (Ward and Hohmann, 1988), with $\tilde{\mathbf{Q}}=\left(\tilde{E}_{x}, \tilde{E}_{y}, \tilde{H}_{y},-\tilde{H}_{x}\right)^{T}$ being the horizontal electric and magnetic field components. By performing the decomposition in the frequency-wavenumber domain, we assume that the medium parameters are laterally invariant at the receiver level, thus at the level where the Fourier transform was computed. There are no restrictions concerning the medium above and below the receiver level, but the separation of TM- and TE-mode is restricted to the receiver level for any general medium. In other words, for a medium featuring a 3D subsurface structure, the separation between the two modes exists only at the receiver level, whereas for a medium consisting of horizontal layers, the separation between the modes is valid for the complete medium because then the TM- and the TE-mode are decoupled (e.g., Appendix A; Chave and Cox, 1982; Kong, 1986; Ward and Hohmann, 1988; Andréis and MacGregor, 2008).

\section{Retrieval of the reflection response}

We split the vector $\tilde{\mathbf{P}}$ in a downward decaying part $\tilde{\mathbf{P}}^{+}$and in an upward decaying part $\tilde{\mathbf{P}}^{-}$. After a discrete Fourier transformation to the frequency-space domain, the two parts can be related to each other by the reflection response of the subsurface $\hat{\mathbf{R}}_{\mathbf{0}}$ as follows

$$
\left(\begin{array}{c}
\hat{\mathbf{P}}^{\mathrm{TM},-} \\
\hat{\mathbf{P}}^{\mathrm{TE},-}
\end{array}\right)=\left(\begin{array}{cc}
\hat{\mathbf{R}}_{\mathbf{0}}^{\mathrm{TM}, \mathrm{TM}} & \hat{\mathbf{R}}_{\mathbf{0}}^{\mathrm{TM}, \mathrm{TE}} \\
\hat{\mathbf{R}}_{\mathbf{0}}^{\mathrm{TE}, \mathrm{TM}} & \hat{\mathbf{R}}_{\mathbf{0}}^{\mathrm{TE}, \mathrm{TE}}
\end{array}\right)\left(\begin{array}{c}
\hat{\mathbf{P}}^{\mathrm{TM},+} \\
\hat{\mathbf{P}}^{\mathrm{TE},+}
\end{array}\right)
$$

where the upward-decaying TM-mode and TE-mode parts, $\hat{\mathbf{P}}^{\text {TM,- }}$ and $\hat{\mathbf{P}}^{\mathbf{T E},-}$, as well as the downward-decaying TM-mode and TE-mode parts, $\hat{\mathbf{P}}^{\mathbf{T M},+}$ and $\hat{\mathbf{P}}^{\mathbf{T E},+}$, are matrices with each column containing the receiver array for one source position and each row containing all the source positions for one receiver position (Berkhout, 1982). The submatrices of the reflection response $\hat{\mathbf{R}}_{\mathbf{0}}$ are matrices set up in a similar way: Each column contains the receiver array for one redatumed source located at the receiver level and vice versa for the rows. The subscript 0 in the reflection response indicates that $\hat{\mathbf{R}}_{\mathbf{0}}$ gives the reflection response of the medium below the receivers whereas the medium above the receivers is homogenized. In other words, $\hat{\mathbf{R}}_{\mathbf{0}}$ does not contain any reflections from above the receiver level. The two superscripts of the submatrices of the reflection response indicate the mode of the receiver and the source, respectively. The reflection response of the subsur- face can be retrieved from equation 3 by inversion of the downward decaying field. This process is called interferometry by MDD.

Assuming a laterally invariant medium, equation 3 can be written in the frequency-wavenumber domain as a set of scalar equations

$$
\begin{aligned}
\tilde{P}^{\mathrm{TM},-}\left(k_{x}, k_{y}, z_{1}, \omega\right)= & \tilde{R}_{0}^{\mathrm{TM}, \mathrm{TM}}\left(k_{x}, k_{y}, z_{1}, \omega\right) \tilde{P}^{\mathrm{TM},+}\left(k_{x}, k_{y}, z_{1}, \omega\right) \\
& +\tilde{R}_{0}^{\mathrm{TM}, \mathrm{TE}}\left(k_{x}, k_{y}, z_{1}, \omega\right) \tilde{P}^{\mathrm{TE},+}\left(k_{x}, k_{y}, z_{1}, \omega\right), \\
\tilde{P}^{\mathrm{TE},-}\left(k_{x}, k_{y}, z_{1}, \omega\right)= & \tilde{R}_{0}^{\mathrm{TE}, \mathrm{TM}}\left(k_{x}, k_{y}, z_{1}, \omega\right) \tilde{P}^{\mathrm{TM},+}\left(k_{x}, k_{y}, z_{1}, \omega\right) \\
& +\tilde{R}_{0}^{\mathrm{TE}, \mathrm{TE}}\left(k_{x}, k_{y}, z_{1}, \omega\right) \tilde{P}^{\mathrm{TE},+}\left(k_{x}, k_{y}, z_{1}, \omega\right) .
\end{aligned}
$$

Because we assumed a laterally invariant medium, the TM-mode and the TE-mode are decoupled as mentioned before (e.g., Appendix A; Chave and Cox, 1982; Kong, 1986; Ward and Hohmann, 1988; Andréis and MacGregor, 2008). This means that a downward-decaying TM-mode signal can only be reflected as an upward-decaying TM-mode signal and transmitted as a downward-decaying TM-mode signal. The same is valid for a TE-mode signal. Thus, modeconversions, i.e., the upward-decaying TM-mode generated by the downward-decaying TE-mode, and vice versa, do not exist. Therefore, the cross-terms $\tilde{R}_{0}^{\mathrm{TM}, \mathrm{TE}}$ and $\tilde{R}_{0}^{\mathrm{TE}, \mathrm{TM}}$ vanish. This simplifies equations 4 and 5 as follows

$\tilde{P}^{\mathrm{TM},-}\left(k_{x}, k_{y}, z_{1}, \omega\right)=\tilde{R}_{0}^{\mathrm{TM}}\left(k_{x}, k_{y}, z_{1}, \omega\right) \tilde{P}^{\mathrm{TM},+}\left(k_{x}, k_{y}, z_{1}, \omega\right)$,

$\tilde{P}^{\mathrm{TE},-}\left(k_{x}, k_{y}, z_{1}, \omega\right)=\tilde{R}_{0}^{\mathrm{TE}}\left(k_{x}, k_{y}, z_{1}, \omega\right) \tilde{P}^{\mathrm{TE},+}\left(k_{x}, k_{y}, z_{1}, \omega\right)$.

The double superscripts of the reflection response have been merged into one superscript because they are equal anyway in the absence of mode-conversions. In equations 6 and 7, the matrix multiplication of equation 3 has become a simple elementwise multiplication. Hence, by division of the upward-decaying field by the downward decaying field in the frequency-wavenumber domain, the reflection response of the subsurface can be retrieved, thus avoiding a matrix inversion. As mentioned before, solving for the reflection response in the frequency-wavenumber domain assumes a laterally invariant medium, i.e., a layered earth. In case the medium is varying laterally, the reflection response needs to be retrieved in the frequency-space domain by inverting equation 3 . Note that the components of the vectors in equation 3 vary between the different cases of interferometry by MDD presented in the next sections. The precise equations are given in the corresponding sections.

\section{MODEL}

Throughout the paper, we use the same subsurface model to create the synthetic data. We chose a 1D subsurface model to facilitate the comparison between interferometry algorithms implemented in the space domain and in the wavenumber domain because the latter requires a layered-earth model. The model consists of a half-space of air, a shallow water-layer of $200 \mathrm{~m}$ thickness and a layered halfspace of sediments, which is intersected by a $50-\mathrm{m}$ thick reservoir layer at $1-\mathrm{km}$ depth below the ocean bottom. The according conductivity values and the depth of the interfaces are given in Figure 1. The receivers are located at the ocean bottom (white triangles) 
recording the four horizontal components of the electromagnetic field $E_{x}, E_{y}, H_{x}$, and $H_{y}$. The source, an antenna emitting an electric field, is $50 \mathrm{~m}$ above the ocean bottom (located in the dashed plane). The frequency of the source signal is $0.5 \mathrm{~Hz}$. The data are modeled in the frequency-wavenumber domain using an analytical code. After a numerical Fourier transform, the data are regridded on a sparser grid with a receiver separation of $640 \mathrm{~m}$ in the inline and crossline directions and with a maximum offset of $20 \mathrm{~km}$ before further processing.

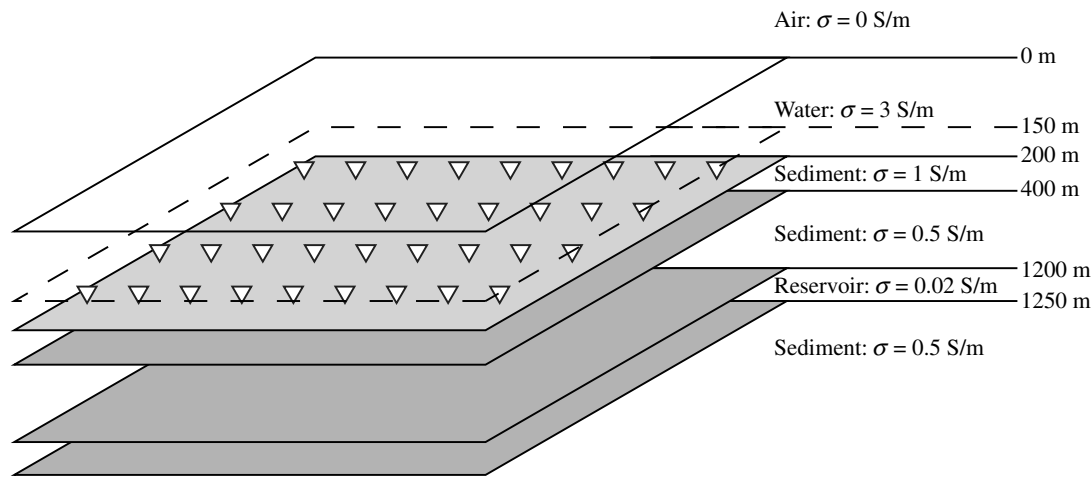

Figure 1. Model used for synthetic data. Transparent interface is the sea surface, light gray interface the sea bottom. All other interfaces in the subsurface are colored dark gray. The source is located $50 \mathrm{~m}$ above the sea bottom in the plane indicated by the dashed line. Note that the conductivity does not change across the dashed plane. The receivers are located at the sea bottom (white triangles). a)

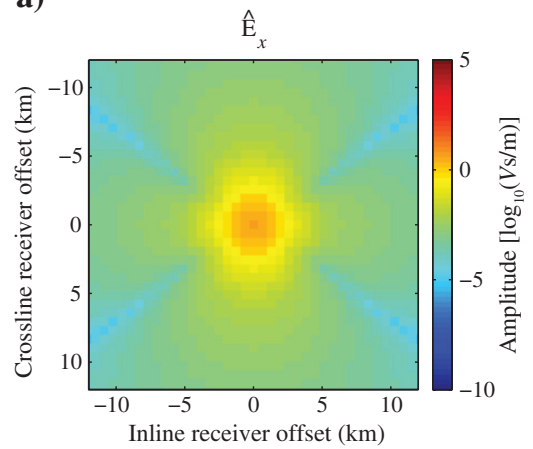

c)

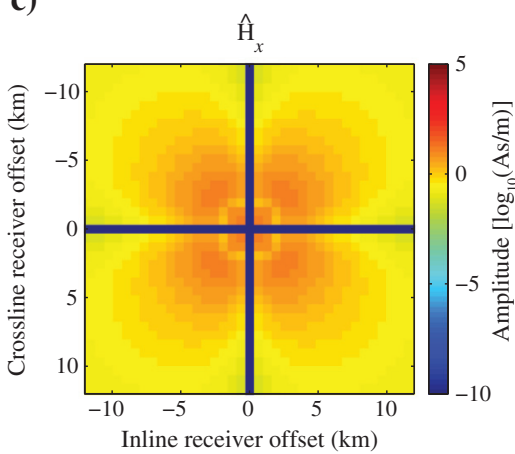

b)

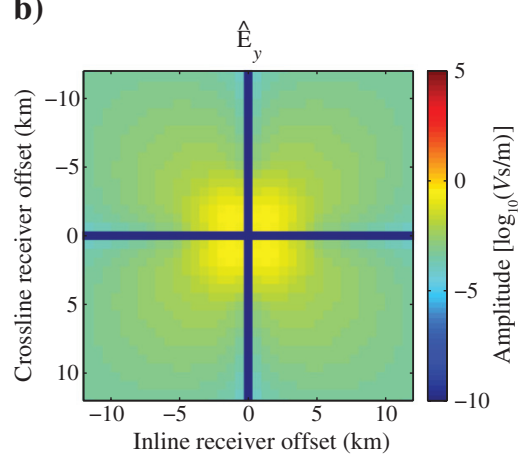

d)

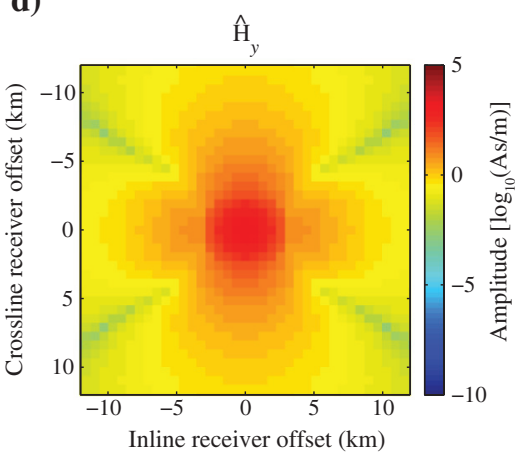

Figure 2. The four horizontal components of the electromagnetic field in the frequencyspace domain for a receiver spacing of $640 \mathrm{~m}$ after the synthetic aperture source has been created: (a) $E_{x}$, (b) $E_{y}$, (c) $H_{x}$, and (d) $H_{y}$.

\section{D INTERFEROMETRY IN THE FREQUENCY- WAVENUMBER DOMAIN}

For 3D interferometry, data on a grid and recordings of all four weighting function of equation 1 . The length of the synthetic aperture source $l$ is chosen as $5 \mathrm{~km}$ and the parameter $\nu$ equal to 5 . We implement the synthetic aperture source in the wavenumber domain for efficiency reasons. The source is oriented in the inline direction and the receiver spacing distance is $640 \mathrm{~m}$ in both horizontal directions. For creating the synthetic aperture source, we assumed to have a similar dense source coverage in the inline and the crossline directions. In practice, the crossline source sampling is sparser than the inline source sampling. The effects of sparser source sampling in the crossline direction remain to be investigated, but are beyond the scope of this paper. At zero inline offset or at zero crossline offset relative to the source position, the amplitude of $E_{y}$ and $H_{x}$ vanishes because of symmetry reasons. Due to the relatively sparse sampling, this zero amplitude shows up as thick blue lines in Figure $2 b$ and $2 c$.

With 3D interferometry, four reflection responses can be retrieved: the TM-mode reflection response, the TE-mode reflection response, and two cross-mode reflection responses. If the medium contained lateral variations, the cross-mode reflection responses would be nonzero. In the frequency-wavenumber domain implementation, where we assume a layered medium, the crossterms are zero according to theory, but can be used as a quality check. If they do not vanish, most probably the assumption of a horizontally layered medium is violated and the frequency-wavenumber domain solution is not appropriate. After Fourier-transforming the data presented in Figure 2 to the frequency-wavenumber domain and decomposing the fields in upward and downward decaying fields, the reflection response can be retrieved by solving equation 3 for the matrix of reflection responses. Unfortunately, the illumination from one source component (inline) is insufficient for a proper retrieval of the $3 \mathrm{D}$ multicomponent reflection response. A more straightforward solution is to use equations 6 and 7 to solve for the TM-mode and the TE-mode reflection response independently. However, if one aims to retrieve the cross-mode reflection responses for a quality check, this independent treatment of each mode is not possible.

In this study, we overcome this limitation by including data of the four horizontal electromagnetic field components generated by a source in the crossline direction. Furthermore, we use a formulation that couples all modes instead of dealing with them independently to retrieve the 
cross-mode reflection responses, which are zero according to theory in a layered earth. The forward problem (equation 3) becomes then in the frequency-wavenumber domain

$$
\left(\begin{array}{cc}
\tilde{P}_{x \text {-src }}^{\mathrm{TM},-} & \tilde{P}_{y \text {-src }}^{\mathrm{TM},-} \\
\tilde{P}_{x \text {-src }}^{\mathrm{TE},-} & \tilde{P}_{y \text {-src }}^{\mathrm{TE},-}
\end{array}\right)=\left(\begin{array}{cc}
\tilde{R}_{0}^{\mathrm{TM}, \mathrm{TM}} & \tilde{R}_{0}^{\mathrm{TM}, \mathrm{TE}} \\
\tilde{R}_{0}^{\mathrm{TE}, \mathrm{TM}} & \tilde{R}_{0}^{\mathrm{TE}, \mathrm{TE}}
\end{array}\right)\left(\begin{array}{cc}
\tilde{P}_{x \text {-src }}^{\mathrm{TM},+} & \tilde{P}_{y \text {-src }}^{\mathrm{TM}+} \\
\tilde{P}_{x \text {-src }}^{\mathrm{TE},+} & \tilde{P}_{y \text {-src }}^{\mathrm{TE},+}
\end{array}\right),
$$

where the subscripts $x$-src and $y$-src in the decomposed fields define the inline and crossline orientation of the source, respectively. All other superscripts and subscripts are the same as before. Note that all quantities are scalars in equation 8 whereas they were matrices in equation 3. The reason is that, in the frequency-wavenumber domain, the reflection response is retrieved separately for each wavenumber.

Thus, equation 8 can be solved for the matrix of reflection responses for each wavenumber separately by inverting the $2 \times 2$ matrix of the downward-decaying field

$$
\begin{aligned}
& \left(\begin{array}{ll}
\tilde{R}_{0}^{\mathrm{TM}, \mathrm{TM}} & \tilde{R}_{0}^{\mathrm{TM}, \mathrm{TE}} \\
\tilde{R}_{0}^{\mathrm{TE}, \mathrm{TM}} & \tilde{R}_{0}^{\mathrm{TE}, \mathrm{TE}}
\end{array}\right)=\left(\begin{array}{cc}
\tilde{P}_{x \text {-src }}^{\mathrm{TM},-} & \tilde{P}_{y \text {-src }}^{\mathrm{TM},-} \\
\tilde{P}_{x \text {-src }}^{\mathrm{TE},-} & \tilde{P}_{y \text {-src }}^{\mathrm{TE},-}
\end{array}\right) \\
& \times\left[\left(\begin{array}{cc}
\tilde{P}_{x \text {-src }}^{\mathrm{TM},+} & \tilde{P}_{y \text {-src }}^{\mathrm{TM},+} \\
\tilde{P}_{x \text {-src }}^{\mathrm{TE},+} & \tilde{P}_{y \text {-src }}^{\mathrm{TE},+}
\end{array}\right)+\varepsilon^{2}\left(\begin{array}{ll}
1 & 0 \\
0 & 1
\end{array}\right)\right]^{-1},
\end{aligned}
$$

where $\varepsilon$ is a stabilization parameter. Although it would be possible to choose a different stabilization parameter for each wavenumber, we use the same for the complete wavenumber spectrum. Three-dimensional interferometry in the frequency-wavenumber domain requires the medium to be laterally invariant. The benefit of the frequency-wavenumber domain solution is that we require only one source location. Furthermore, the inversion problem reduces to a simple $2 \times 2$ matrix inversion. For any general medium, 3D interferometry with several source locations involving a large matrix inversion needs to be applied in the frequency-space domain, which is discussed in the next section.

The reflection responses retrieved with equation 9 miss one datapoint at $k_{x}=k_{y}=0$, i.e., the reflection response for vertical incidence, because that wavenumber component of the field is not excited by a finite source. To fill the gap, this one point is given the value of its neighboring point, i.e., the smallest wavenumber available in the $k_{x}$-direction. The amplitude of the resulting retrieved reflection responses in the frequency-wavenumber domain for the TM-mode and the TE-mode are shown in Figure $3 \mathrm{a}$ and 3 b. The complete wavenumber range available for a receiver-sampling distance of $640 \mathrm{~m}$ is shown. The retrieved reflection responses are radially symmetric and agree reasonably well compared with the directly modeled reflection responses shown in Figure $3 \mathrm{c}$ and $3 \mathrm{~d}$, except for edge artifacts that disturb the signal at the largest wavenumbers. These artifacts can be damped by using a larger stabilization parameter at these wavenumbers, but the improvement is only cosmetic because the retrieved reflection response for these wavenumbers is minimized without producing the correct values. The relative error between retrieved and modeled reflection responses is shown in Figure $3 \mathrm{e}$ and $3 \mathrm{f}$. In the TM-mode as well as in the TE-mode, the relative error never exceeds $3 \%$ except at high wavenumbers (i.e., $>0.65 \mathrm{~km}^{-1}$ for the TM-mode and $>0.6 \mathrm{~km}^{-1}$ for the TE-mode). The reflection responses for mode conversions are correctly retrieved to be numerically zero (not shown), i.e., the mean of the cross-mode reflection responses is more than three orders of magnitude smaller than the mean of the TM-mode reflection response (excluding the edge artifacts). This also indicates a proper retrieval of the multicomponent reflection response.

Before the Fourier transform to the frequency-space domain is carried out, a taper is applied to damp the unstable signal close to the Nyquist wavenumbers. The retrieved and modeled reflection responses in the frequency-space domain are shown in Figure $4 \mathrm{a}-4 \mathrm{~d}$. Note that a)

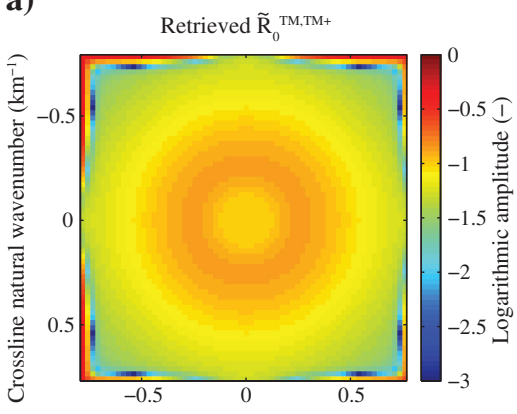

Inline natural wavenumber $\left(\mathrm{km}^{-1}\right)$

c) $\quad$ Modeled $\widetilde{\mathrm{R}}_{0}^{\mathrm{TM}, \mathrm{TM}+}$

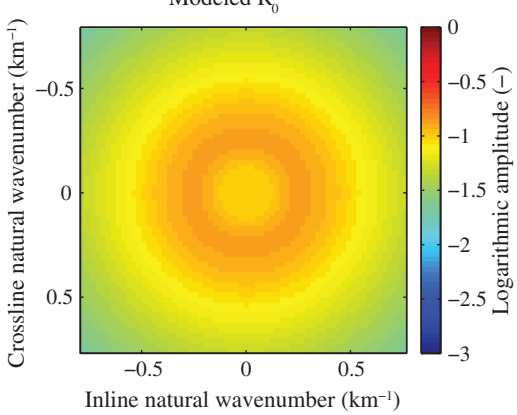

e)

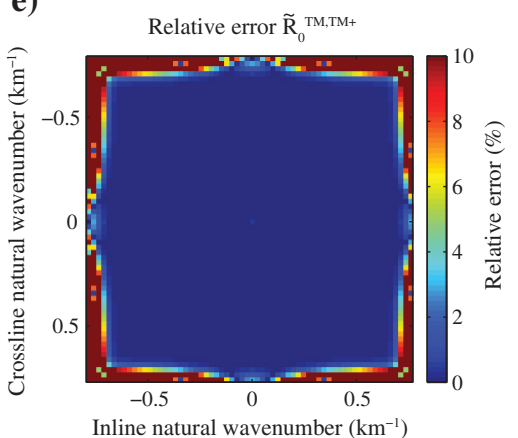

b)

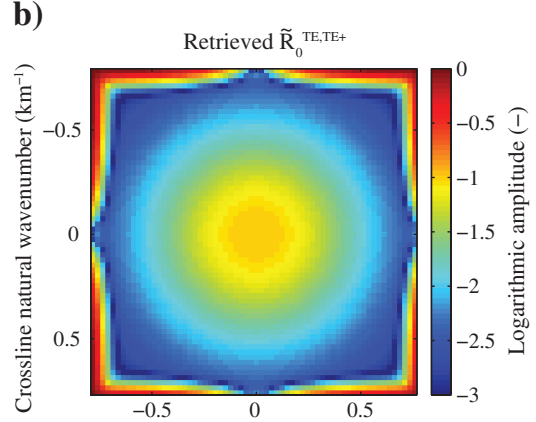

Inline natural wavenumber $\left(\mathrm{km}^{-1}\right)$

d)

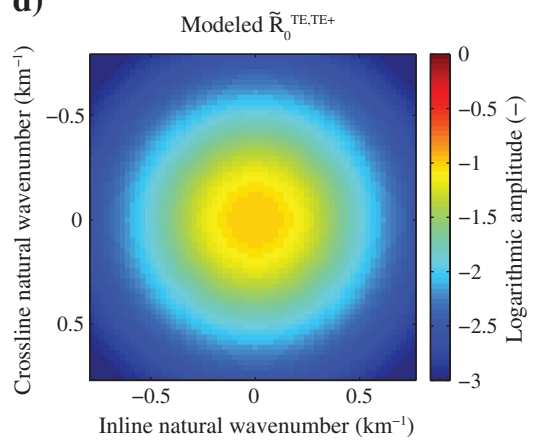

f)

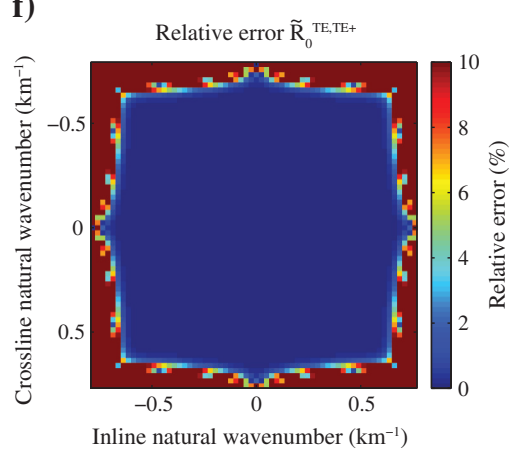

Figure 3. The reflection responses retrieved and plotted in the frequency-wavenumber domain: (a) retrieved TM-mode, (b) retrieved TE-mode, (c) modeled TM-mode, (d) modeled TE-mode, (e) relative error TM-mode, and (f) relative error TE-mode. 
the modeled reflection response has been bandlimited to the same bandwidth as the reflection response retrieved with a receiver spacing of $640 \mathrm{~m}$. The corresponding relative error plots are shown in Figure $4 \mathrm{e}$ and $4 \mathrm{f}$. The reflection responses are retrieved well, apart from instabilities confined to the zero inline and zero crossline offset. These artifacts are introduced in the Fourier transform because the reflection response is aliased at a receiver spacing of $640 \mathrm{~m}$. The same artifacts appear also in the bandlimited modeled reflection response, which confirms that aliasing is the cause of the artifacts. Only a denser receiver spacing will avoid these artifacts. In the diagonal direction $(x= \pm y)$, no aliasing takes place due to the larger radial wavenumber range. Because the reflection responses are radial functions in a layered medium, no information is lost in that case. The relative error plots show errors smaller than $10 \%$ for radial distances from the source smaller than $10 \mathrm{~km}$. At larger radial distances, the relative error increases beyond $10 \%$, but at those offsets, the signal has decayed already over four orders of magnitude and is, therefore, rather small. Due to the small amplitude of the field at this offset range, even very small absolute errors can show up on the relative error plots with a huge magnitude. Therefore, we neglect these errors. Considering the large receiver-sampling distances used, this retrieval is considered very good. Smaller receiversampling distances will lead to an even better retrieval.

\section{D INTERFEROMETRY IN THE FREQUENCY-SPACE DOMAIN}

For frequency-space domain 3D interferometry, no limitations about the medium apply, but a set of sources is required to retrieve the reflection response and not just one as for the frequencywavenumber domain interferometry. To perform 3D interferometry by MDD in the frequency-space domain, we rewrite equation 8 in the frequency-space domain. In that case, the elements of the matrices become matrices themselves

$$
\left(\begin{array}{cc}
\hat{\mathbf{P}}_{x \text {-src }}^{\mathrm{TM},-} & \hat{\mathbf{P}}_{y \text {-src }}^{\mathrm{TM},-} \\
\hat{\mathbf{P}}_{x \text {-src }}^{\mathrm{TE},-} & \hat{\mathbf{P}}_{y \text {-src }}^{\mathrm{TE},-}
\end{array}\right)=\left(\begin{array}{cc}
\hat{\mathbf{R}}_{0}^{\mathrm{TM}, \mathbf{T M}} & \hat{\mathbf{R}}_{0}^{\mathrm{TM}, \mathbf{T E}} \\
\hat{\mathbf{R}}_{0}^{\mathrm{TE}, \mathbf{T M}} & \hat{\mathbf{R}}_{0}^{\mathrm{TE}, \mathbf{T E}}
\end{array}\right)\left(\begin{array}{cc}
\hat{\mathbf{P}}_{x \text {-src }}^{\mathrm{TM}}+ & \hat{\mathbf{P}}_{y \text {-src }}^{\mathrm{TM},+} \\
\hat{\mathbf{P}}_{x \text {-src }}^{\text {TE },+} & \hat{\mathbf{P}}_{y \text {-src }}^{\text {TE },+}
\end{array}\right),
$$

a)

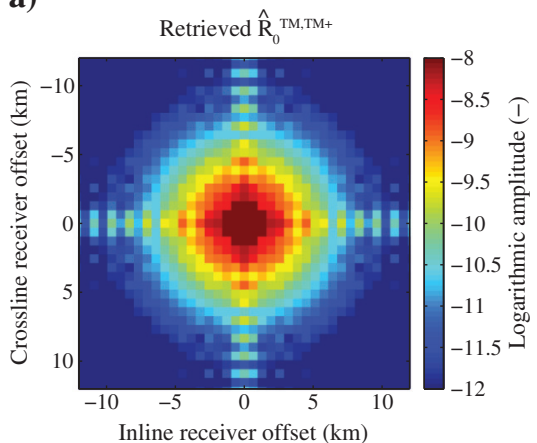

c)

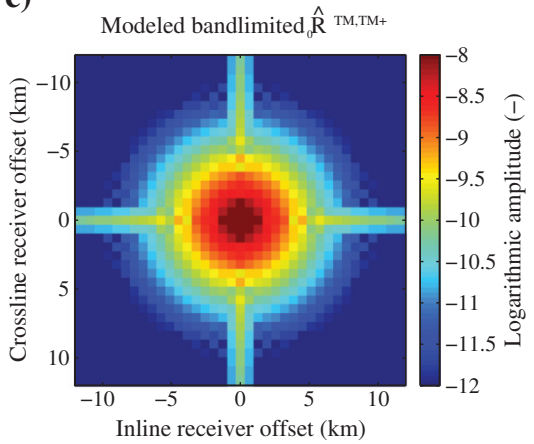

e)

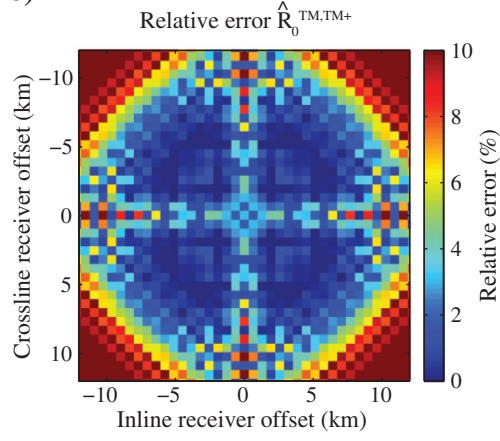

b)

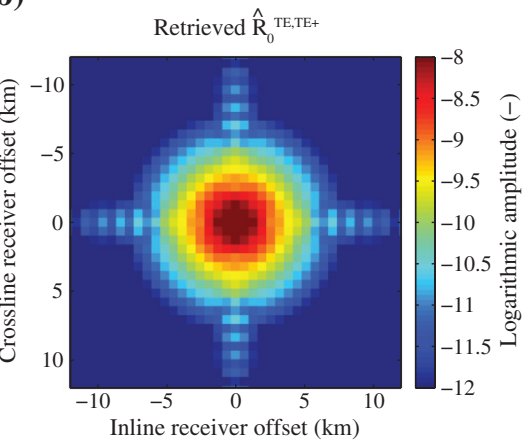

d)

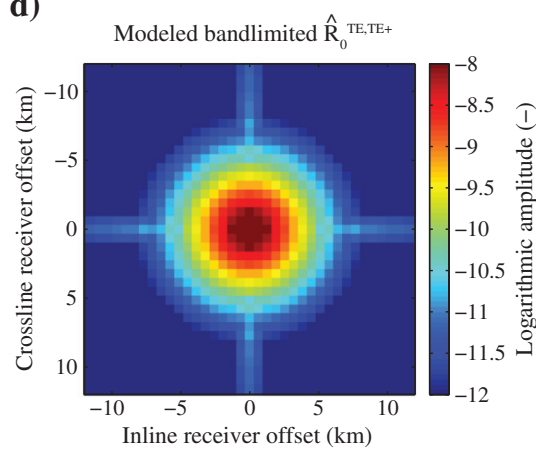

f)

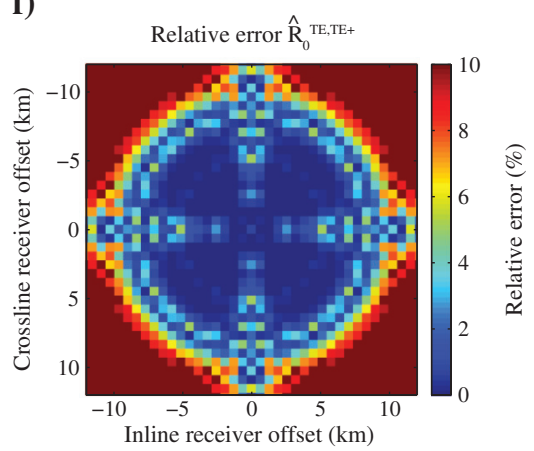

Figure 4. Same data as in Figure 3 but plotted in the frequency-space domain.

where the matrices $\hat{\mathbf{P}}$ contain the decomposed fields for all receiver positions for one source position along their columns and vice versa for the rows. The matrices $\hat{\mathbf{R}}$ are structured in a similar way, but with the source positions replaced by redatumed source positions. Because a source is redatumed at every receiver position, the matrices $\hat{\mathbf{R}}$ are square. Note that the grid of receivers and the grid of sources is represented in one column or one row, respectively, to avoid tensors of higher order than two (Kinneging et al., 1989). The disadvantage of this approach is that the matrices are large. For example, for a receiver grid of $64 \times 64$ receivers, as it is used in this example, one row of the matrix $\hat{\mathbf{P}}_{x \text {-src }}^{\mathrm{TM},+}$ contains $64^{2}=4096$ elements. To create a similar source coverage as is simulated by solving equation 8 in the frequency-wavenumber domain, a grid of $(2 \cdot 64-1)^{2}=16129$ sources is necessary. Consequently, matrix $\hat{\mathbf{P}}_{x \text {-src }}^{\mathrm{TM}+}$ ends up having $4096 \times 16,129$ elements. If the complex data are stored as 64-bit floating-point digits, approximately one gigabyte of memory is required to set up the matrix $\hat{\mathbf{P}}_{x \text {-src }}^{\mathrm{TM}+}$. Four times more is necessary to keep the complete downward decaying field in memory. To have a slightly more realistic example, we omit source positions far away from the receiver grid. In total, 7744 sources are included in our example data set.

Although - in the frequency-wavenumber domain case - the matrix that needs to be inverted is always square, with the size depending only on the components (a $2 \times 2$ matrix), it is not square in the frequency-space domain because the size of the matrix depends on the number of receivers and the number of sources. Therefore, we use the following least-squares equation to retrieve the reflection response 


$$
\begin{aligned}
& \left(\begin{array}{ll}
\hat{\mathbf{R}}_{\mathbf{0}}^{\mathrm{TM}, \mathrm{TM}} & \hat{\mathbf{R}}_{\mathbf{0}}^{\mathrm{TM}, \mathrm{TE}} \\
\hat{\mathbf{R}}_{\mathbf{0}}^{\mathrm{TE}, \mathrm{TM}} & \hat{\mathbf{R}}_{\mathbf{0}}^{\mathrm{TE}, \mathrm{TE}}
\end{array}\right)=\left(\begin{array}{ll}
\hat{\mathbf{P}}_{x \text {-src }}^{\mathrm{TM},-} & \hat{\mathbf{P}}_{y \text {-src }}^{\mathrm{TM},-} \\
\hat{\mathbf{P}}_{x \text {-src }}^{\mathrm{TE},-} & \hat{\mathbf{P}}_{y \text {-src }}^{\mathrm{TE},-}
\end{array}\right)\left(\begin{array}{ll}
\hat{\mathbf{P}}_{x \text {-src }}^{\mathrm{TM},+} & \hat{\mathbf{P}}_{y \text {-src }}^{\mathrm{TM},+} \\
\hat{\mathbf{P}}_{x \text {-src }}^{\mathrm{TE},+} & \hat{\mathbf{P}}_{y \text {-src }}^{\mathrm{TE},+}
\end{array}\right) \\
& \times\left[\left(\begin{array}{cc}
\hat{\mathbf{P}}_{x-\text { src }}^{\mathrm{TM},+} & \hat{\mathbf{P}}_{y \text {-src }}^{\mathrm{TM},+} \\
\hat{\mathbf{P}}_{x \text {-src }}^{\mathrm{TE},+} & \hat{\mathbf{P}}_{y \text {-src }}^{\mathrm{TE},+}
\end{array}\right)\left(\begin{array}{cc}
\hat{\mathbf{P}}_{x \text {-src }}^{\mathrm{TM},+} & \hat{\mathbf{P}}_{y \text {-src }}^{\mathrm{TM},+} \\
\hat{\mathbf{P}}_{x \text {-src }}^{\mathrm{TE},+} & \hat{\mathbf{P}}_{y \text {-src }}^{\mathrm{TT},+}
\end{array}\right)^{\dagger}+\varepsilon^{2} f_{x} \mathbf{I}\right]^{-1} .
\end{aligned}
$$

The scaling factor $f_{x}$ is the mean of the absolute value of the diagonal elements of the crosscorrelation of the downward decaying field with itself. All other quantities are as defined previously.

The retrieved reflection responses are shown in Figure 5a and $5 \mathrm{~b}$, the directly modeled bandlimited reflection responses are reproduced from Figure 4 in Figure $5 \mathrm{c}$ and $5 \mathrm{~d}$ and the relative error between the two is shown in Figure 5e and 5f. The retrieval is quite accurate, with a relative error smaller than $10 \%$ in most of the large amplitude area. The cross-mode reflection responses show some signal around zero-offset, but it is more than two orders of magnitude smaller than the signal of the TM-mode reflection response at the same location. Therefore, we consider the cross-mode reflection responses, also in this case, properly retrieved as numerically zero (not shown). The solution depends strongly on the stabilization parameter $\varepsilon$. Because the retrieval of the reflection response in the frequency-space domain is much more expensive than in the frequency-wavenumber domain, only a limited amount of values for the stabilization parameter could be tested. A better choice may lead to a more accurate solution with a smaller relative error. The TM-mode especially could be improved. Still, the frequency-space domain solution is slightly worse than the frequency-wavenumber solution. These artifacts may be caused in the decomposition by improperly sampled fields for sources close to the edge of the receiver array. The decomposition, which is carried out per source position, assumes that, for each source, enough receivers on all sides of the source are available. This assumption is not fulfilled for sources toward the edge or even outside the receiver array and, therefore, introduces artifacts. Another reason for the noisier retrieval of the reflection response may be found in the difference of the stabilization parameter of equations 9 and 11. Although in the frequencywavenumber domain, unstable wavenumbers are stabilized, the field at unstable spatial locations is stabilized in the frequency-space domain. The wavenumber-domain stabilization may be closer to the actual physics and, therefore, deliver a less noisy result. Note that this algorithm can be applied to any medium, although the example shown here is a layered-earth situation.

As an alternative to the least-squares solution, a conjugate-gradient scheme may be considered. This would be a way to work around the strong dependency on the stabilization parameter. Furthermore, a conjugate-gradient scheme can be implemented in such a way that the com- plete matrix to invert does not need to be kept in memory. Each iteration can be divided into steps that just require one row and one column of that matrix. One iteration is completed, once all the rows and columns have been processed. This allows to process the current acquisition size on a smaller computer or use even a larger acquisition grid. Furthermore, this algorithm is suited for parallel computing.

\section{RESERVOIR DETECTABILITY AFTER 3D INTERFEROMETRY IN THE FREQUENCY- WAVENUMBER DOMAIN}

As mentioned earlier, the retrieved reflection response suppresses the direct field and the airwave. Therefore, the reservoir should be better detectable. To investigate this, we computed the normalized amplitude of the original electromagnetic fields as well as of the reflection responses retrieved in the frequency-wavenumber domain. Normalized amplitude means that the amplitude of a data a)

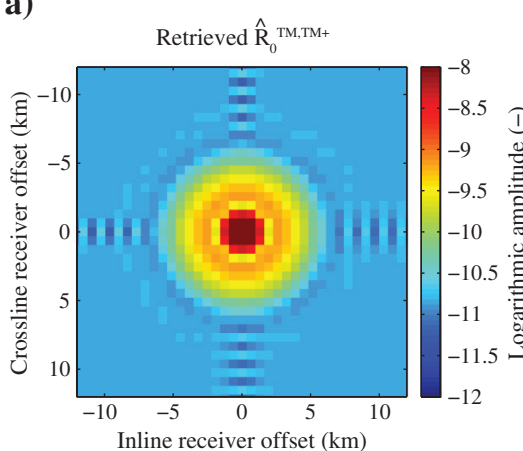

c)

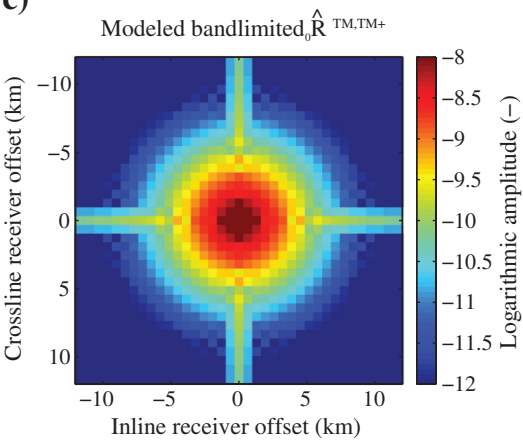

e)

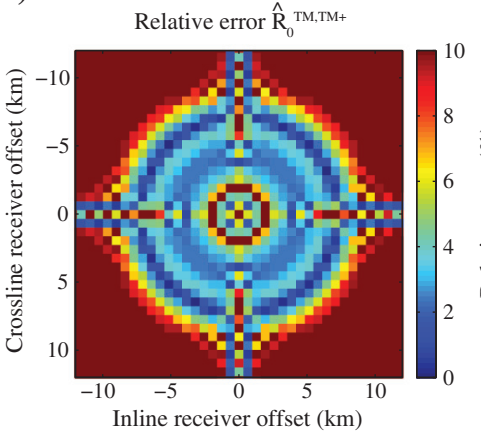

b)

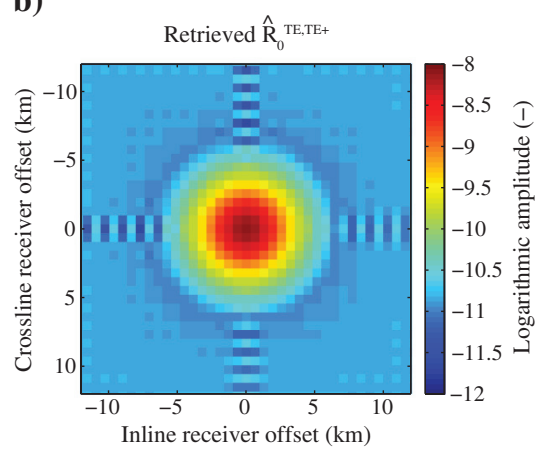

d)

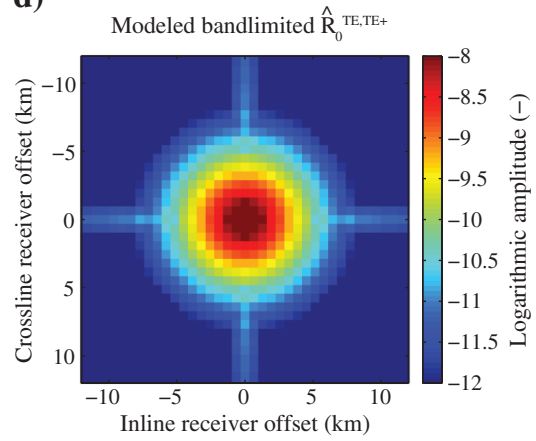

f)

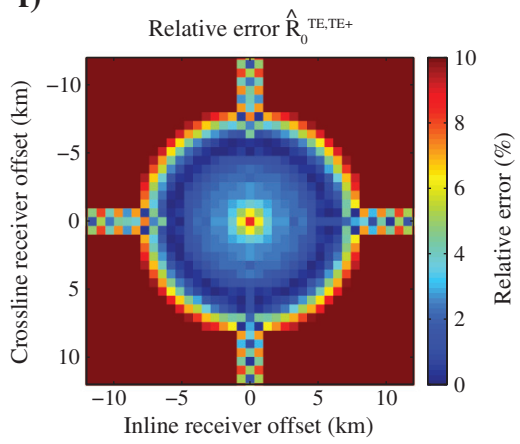

Figure 5. The reflection responses retrieved and plotted in the frequency-space domain: (a) retrieved TM-mode, (b) retrieved TE-mode, (c) modeled TM-mode, (d) modeled TE-mode, (e) relative error TM-mode, and (f) relative error TE-mode. 
set is normalized with a corresponding data set for a situation without a reservoir, i.e., Figure 1 without the 50-m thick layer of $0.02 \mathrm{~S} / \mathrm{m}$ conductivity at $1200 \mathrm{~m}$ depth. We use here this straightforward normalized amplitude as a measure for the detectability of the reservoir because it is a standard procedure in CSEM. However, it may be possible to extract more information about the reservoir using an alternative definition of the normalized amplitude as described by Mittet (2008).

The normalized amplitude of the four horizontal electromagnetic fields for an inline source as well as the TM-mode and the TE-mode reflection responses are shown in Figure 6. A normalized amplitude of around one means that the reservoir cannot or can only barely be seen. Anything significantly larger than one represents a strong imprint of the reservoir. Note that the color scale is cut at normalized amplitudes of four. The normalized amplitude of the four horizontal electromagnetic field components are at most offsets only slightly a)

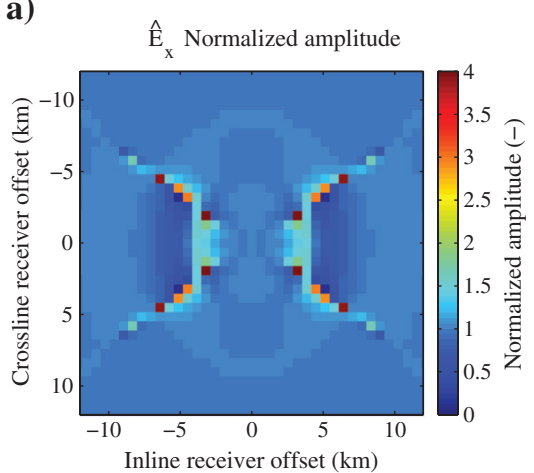

c)

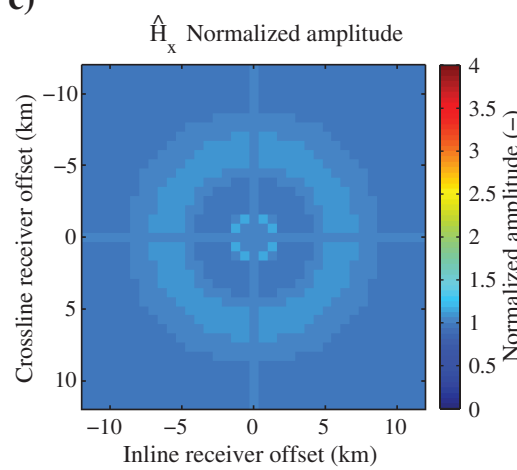

e)

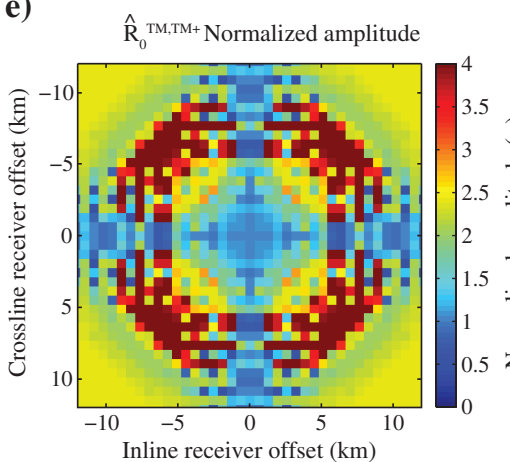

b)

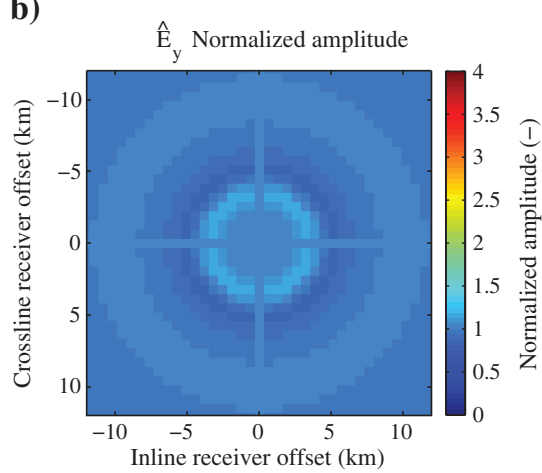

d)

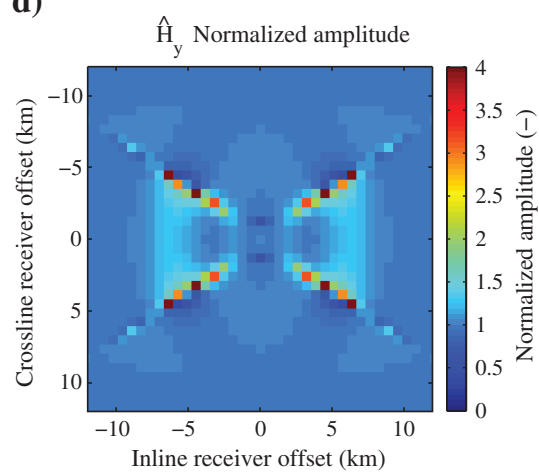

f)

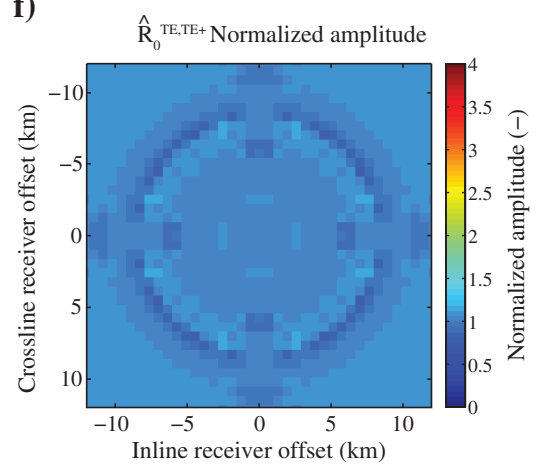

Figure 6. Reservoir response, i.e., the amplitude of the data normalized with the amplitude of the same data set without a reservoir, for (a) $E_{x}$, (b) $E_{y}$, (c) $H_{x}$, (d) $H_{y}$, (e) $R_{0}^{\mathrm{TM}, \mathrm{TM}}$, and (f) $R_{0}^{\mathrm{TE}, \mathrm{TE}}$. above one, indicating that the reservoir leaves only a small imprint in the data. However, in $E_{x}$ and $H_{y}$ (Figure 6a and 6d) some datapoints show a normalized amplitude up to 12 . These datapoints lie on or close to zero-crossings of the field (compare with Figure 2). Therefore, we consider these large normalized amplitudes to be numerical artifacts.

In contrast, the TM-mode reflection response (Figure 6e) shows a normalized amplitude larger than two at all offsets. Over a large area, the normalized amplitude is even larger than four. The best detectability comes from receivers on diagonal locations on the receiver grid because those receivers suffer the least from aliasing artifacts shown in Figure 4. Figure 6e indicates a strong imprint of the reservoir in the TM-mode reflection response. The situation is completely different for the TE-mode reflection response (Figure 6f) which shows similar normalized amplitude values as the original electromagnetic fields. Consequently, the TM-mode is much more sensitive to the reservoir than the TE-mode. This is a well-known fact described, for example, by Eidesmo et al. (2002).

\section{PARAMETER DEPENDENCY OF 3D INTERFEROMETRY}

When applying interferometry, the choice of two parameters is crucial. These parameters are, first, the conductivity $\sigma$ of the subsurface at the receiver level and, second, the stabilization parameter $\varepsilon$. In synthetic examples, we know the conductivity $\sigma$ of the seabed where the receivers are located. In reality, one may have some local measurements or one needs to estimate the conductivity, hence the conductivity value $\sigma$ used for the decomposition will be imprecise. To choose the stabilization parameter $\varepsilon$ for the examples shown in this paper, we used an algorithm which minimizes the relative error between the retrieved and the modeled reflection response. In reality, there is no modeled reflection response and, therefore, $\varepsilon$ needs to be chosen by trial and error, making it unlikely to find the optimum value. A way to find a good value for $\varepsilon$ is to start with a rather large $\varepsilon$ which is subsequently decreased until the solution gets unstable. A good choice would be the smallest $\varepsilon$ which gives a stable solution. Inversions based on conjugate gradient schemes or singular value decomposition may be used as an alternative to the least-squares inversion used for interferometry in the frequencyspace domain to avoid a difficult process to find the proper stabilization parameter. Comparable parameters used in these schemes are closer related to the physics of the problem. Still, it is important to know the influence of wrongly chosen or imprecise $\varepsilon$ and $\sigma$ on the retrieved reflection response.

To this end, we retrieved reflection responses for a range of conductivity values $\sigma$ and stabilization parameters $\varepsilon$ using 3D interferometry in the frequency-wavenumber domain (equation 9). For each of these retrieved reflection responses, 
we computed the cumulative relative error $E$ for each mode independently

$$
E^{m, n}(\sigma, \varepsilon)=\frac{\Sigma_{\text {samples }}|| \tilde{R}_{0, \text { ret }}^{m, n}(\sigma, \varepsilon)|-| \tilde{R}_{0, \text { mod }}^{m, n} \|}{\Sigma_{\text {samples }}\left|\tilde{R}_{0, \text { mod }}^{m, n}\right|},
$$

where the superscripts $m$ and $n$ indicate the mode of the receiver and the mode of the redatumed source, respectively. In this study, we only consider situations where $m=n$ because the cross-terms $(m \neq n)$ vanish in a horizontally layered medium. The subscripts "ret" and "mod" refer to retrieved and modeled reflection response, respectively. The sum is taken over a range of samples in the frequency-wavenumber domain. We do not include wavenumbers close to the Nyquist frequency $\left(>0.6 \mathrm{~km}^{-1}\right)$ because they show unreasonably high amplitudes for a vast range of small $\varepsilon$, which would dominate the cumulative relative error function although the retrieval of the reflection response is quite good otherwise. Due to the rather large computational time necessary to retrieve the reflection response in the frequency-space domain, these computations are only possible using the frequency-wavenumber implementation.

Figure 7 shows the cumulative error for the TM-mode and TEmode situation whose retrieved reflection responses for the optimal choice of $\sigma$ and $\varepsilon$ are shown in Figures 3 and 4. If the cumulative relative error is close to zero (dark blue areas) the reflection response is retrieved well and if the cumulative relative error is large (red areas), the retrieved reflection response is far from the correct solution. The correct conductivity $\sigma$ in our situation is $1 \mathrm{~S} / \mathrm{m}$. For both modes, we find an area between $0.9 \mathrm{~S} / \mathrm{m}$ and $1.1 \mathrm{~S} / \mathrm{m}$ which is dark blue. In other words, the conductivity needs to be known with a precision of $\pm 0.1 \mathrm{~S} / \mathrm{m}$ of the correct value in our simulation. The plots in Figure 7 are asymmetric relative to the conductivity axis, which indicates that overestimating the conductivity leads to a larger error than underestimating it.

The stabilization parameter $\varepsilon$ hardly affects the solution of interferometry in the frequency-wavenumber domain as long as it is chosen small enough, i.e., smaller than approximately $10^{2}$. Overly large values of $\varepsilon$, on the other hand, lead to a very small amplitude of the retrieved reflection response and, consequently, the relative cumulative error becomes one (green areas). Although interferometry in $3 \mathrm{D}$ in the frequency-wavenumber domain is stable, 3D interferometry in the frequency-space domain tends to become unstable for small values of $\varepsilon$ (not shown).

\section{DISCUSSION}

Solving for the reflection response in the frequency-wavenumber domain involves a matrix inversion of a $2 \times 2$ matrix because all four components of the reflection response are retrieved. A matrix inversion can be avoided by setting the cross-terms $\tilde{R}_{0}^{\mathrm{TM}, \mathrm{TE}}$ and $\tilde{R}_{0}^{\mathrm{TE}, \mathrm{TM}}$ to zero in equation 8 . They have to be zero because the wavenumber-domain solution assumes a layered earth. Then both sides are multiplied with the complex conjugate and transposed matrix containing the downward decaying field. This gives two scalar equations for the TM-mode and the TE-mode reflection response

$$
\begin{gathered}
\tilde{R}_{0}^{\mathrm{TM}, \mathrm{TM}}=\frac{\tilde{P}_{x \text {-src }}^{\mathrm{TM},-} \tilde{P}_{x \text {-src }}^{\mathrm{TM},+*}+\tilde{P}_{y \text {-src },-}^{\mathrm{TM}} \tilde{P}_{y \text {-src }}^{\mathrm{TM},+*}}{\tilde{P}_{x \text {-src }}^{\mathrm{TM},+} \tilde{P}_{x \text {-src }}^{\mathrm{TM},+*}+\tilde{P}_{y \text {-src }}^{\mathrm{TM},+} \tilde{P}_{y \text {-src }}^{\mathrm{TM},+*},} \\
\tilde{R}_{0}^{\mathrm{TE}, \mathrm{TE}}=\frac{\tilde{P}_{x \text {-src }}^{\mathrm{TE},-} \tilde{P}_{x \text {-src }}^{\mathrm{TE},+*}+\tilde{P}_{y \text {-src }}^{\mathrm{TE},-} \tilde{P}_{y \text {-src }}^{\mathrm{TE},+*}}{\tilde{P}_{x \text {-src }}^{\mathrm{TE},+} \tilde{P}_{x \text {-src }}^{\mathrm{TE},+*}+\tilde{P}_{y \text {-src }}^{\mathrm{TE},+} \tilde{P}_{y \text {-src }}^{\mathrm{TE},+*}}
\end{gathered}
$$

where the superscript asterisk means complex conjugation. Consequently, in the frequency-wavenumber domain, the reflection response can be retrieved without performing a matrix inversion. Advantages of carrying out the matrix inversion are that in one step the TM-mode as well as the TE-mode reflection response are retrieved and, as mentioned before, the mode-conversions being zero can be used as a quality check.

All the examples shown here are based on noise-free data. Random noise as well as random errors in receiver position and orientation for 2D-interferometry are discussed by Hunziker et al. (2012b). That study showed that even relatively large levels of random noise poses no problem because it averages out when the synthetic aperture source is created. Random positioning and orientation errors limit the bandwidth of the properly retrieved reflection response. Only rather large receiver positioning errors (up to $50 \mathrm{~m}$ ) but already small orientation errors (up to $10^{\circ}$ ) disturb the data significantly. Consequently, receiver positioning errors pose no problem either. Receiver orientation errors can be avoided by using six component receivers and measuring the orientation of the receivers. Subsequently, the data can be rotated virtually to overcome orientation errors. We assume that, in 3D, random noise and random errors in receiver position and orientation affect the data in a similar way as in $2 \mathrm{D}$. a)

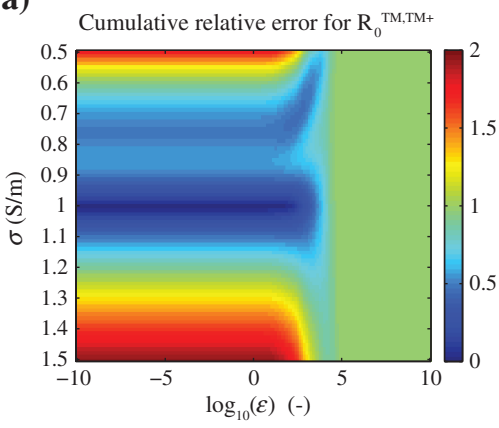

b)

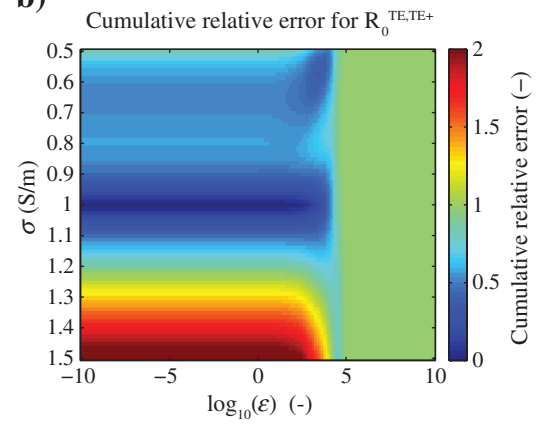

Figure 7. The cumulative error function for different conductivity values $\sigma$ and different stabilization values $\varepsilon$ for (a) the TM-mode and (b) the TE-mode reflection response.

Table 1. Components for the TE- and TM-mode field equations.

\begin{tabular}{lcccccc} 
Mode & $u_{1}$ & $u_{2}$ & $A_{12}$ & $A_{21}$ & $s_{1}$ & $s_{2}$ \\
\hline $\mathrm{TM}$ & $\hat{\mathbf{z}} \cdot\left(i \mathbf{k}_{T} \times \tilde{\mathbf{H}}_{T}\right)$ & $i \mathbf{k}_{T} \cdot \tilde{\mathbf{E}}_{T}$ & $\eta$ & $\zeta+\kappa^{2} / \eta$ & $-i \mathbf{k}_{T} \cdot \tilde{\mathbf{J}}_{T}^{e}$ & $-\kappa^{2} \tilde{\mathbf{J}}_{z}^{e} / \eta-\hat{\mathbf{z}} \cdot\left(i \mathbf{k}_{T} \times \tilde{\mathbf{J}}_{T}^{m}\right)$ \\
$\mathrm{TE}$ & $\hat{\mathbf{z}} \cdot\left(i \mathbf{k}_{T} \times \tilde{\mathbf{E}}_{T}\right)$ & $-i \mathbf{k}_{T} \cdot \tilde{\mathbf{H}}_{T}$ & $\zeta$ & $\eta+\kappa^{2} / \zeta$ & $i \mathbf{k}_{T} \cdot \tilde{\mathbf{J}}_{T}^{m}$ & $\kappa^{2} \tilde{J}_{z}^{m} / \zeta-\hat{\mathbf{z}} \cdot\left(i \mathbf{k}_{T} \times \tilde{\mathbf{J}}_{T}^{e}\right)$ \\
\hline
\end{tabular}




\section{CONCLUSIONS}

Interferometry by MDD applied to CSEM data suppresses any interactions of the signal with the water and the air-water interface (including the airwave). Furthermore, the direct field is suppressed and the source is redatumed to the receiver level. Because interferometry by MDD is a data-driven method, no information about the subsurface or the source position is required except the material parameters of the seabed and the receiver positions and orientations. We applied interferometry by MDD to 3D synthetic data of a layered-earth model in the frequency-wavenumber and in the frequency-space domain.

To retrieve the matrix of reflection responses properly with $3 \mathrm{D}$ interferometry in both domains, data of an inline-oriented source are not sufficient. Data of a crossline-oriented source are also needed. The wavenumber-domain solution requires the medium to be laterally invariant, whereas the space-domain algorithm presented here can be applied to any medium. Considering computational efficiency, the wavenumber-domain algorithm is much faster than the space-domain implementation. Although the process for a data set of $64 \times 64$ receivers takes only a few minutes in the wavenumber domain, it takes up to several days in the space domain (including synthetic aperture and decomposition of all the source positions required) using one $2 \mathrm{GHz}$ AMD Opteron cpu, which is part of a cluster consisting of 32 processors, and a total of $132 \mathrm{~GB}$ of memory.

Interferometry in the frequency-wavenumber domain produces with a relative error of about $2 \%$ in the high amplitude area more accurate results than interferometry in the frequency-space domain, which has a relative error of $3 \%$ to $4 \%$ in the central area. This may not be the case for real data because the assumption made for interferometry in the wavenumber domain (no horizontal variation of the medium) may be unrealistic. However, interferometry in the frequency-space domain requires a set of sources that illuminate the subsurface from as many angles as possible. Sources close to the edge or outside the receiver array are not properly sampled, i.e., the electromagnetic field for these sources is not recorded on all sides of the source for large enough offsets. This introduces artifacts, which finally disturb the retrieved reflection response.

We computed the normalized amplitude of the electromagnetic fields and of the retrieved reflection responses to determine if the detectability of the reservoir is increased after interferometry has been applied. The normalized amplitude of the electromagnetic fields before interferometry are not larger than 1.5 and only at specific offsets (around $4 \mathrm{~km}$ for the inline electric field and around $6 \mathrm{~km}$ for the crossline magnetic field) and at specific angles. This indicates that the reservoir is barely detectable. In contrast, the normalized amplitude of the TM-mode reflection response is at least two on all offsets larger than $2.5 \mathrm{~km}$ but features for a large offset range (between 5 and $8 \mathrm{~km}$ ) values larger than 4 . Furthermore, the detectability of the reservoir is angle-independent excluding aliasing artifacts on certain angles. On the other hand, the TE-mode reflection response does not show sensitivity to the reservoir featuring normalized amplitudes not larger than 1.3. These findings show, first, that interferometry is able to increase the detectability of the reservoir up to a factor of six in our example; second, that the offset range on which the reservoir can be detected increases; and, third, that the reservoir information is mainly in the TM-mode of the electromagnetic field.
For the decomposition, it is necessary to know the conductivity of the seabed. In our situation, the conductivity was required with a precision of $\pm 0.1 \mathrm{~S} / \mathrm{m}$. Overestimating the conductivity has a larger effect than underestimating it. The other crucial parameter in the interferometry scheme is the stabilization parameter. A stabilization parameter that is too large damps the amplitude of the retrieved reflection response. In the 3D frequency-space-domain interferometry, a too-small stabilization parameter introduces instabilities in the retrieved reflection response.

\section{ACKNOWLEDGMENTS}

This research is supported by the Dutch Technology Foundation, the Applied Science division of the Netherlands Organization for Scientific Research, and the Technology Program of the Ministry of Economic Affairs. We thank Rune Mittet, three anonymous reviewers, and the editors for their comments.

\section{APPENDIX A}

\section{MAXWELL'S EQUATIONS DECOMPOSED IN TE-MODE AND TM-MODE FIELDS FOR A LAYERED MODEL}

In this appendix, we show that the TM-mode and the TE-mode are separable in a layered-earth model. This appendix has been added because there seems to be much controversy about this issue as we understand from several anonymous reviewers.

Vector notation $\mathbf{E}=\left\{E_{x}, E_{y}, E_{z}\right\}$ is used and horizontal components of a vector field are denoted $\mathbf{E}_{T}=\left\{E_{x}, E_{y}, 0\right\}$. The electric and magnetic field vectors are denoted $\mathbf{E}, \mathbf{H}$, the medium parameters are electric conductivity $\sigma$ and permittivity $\varepsilon$ and magnetic permeability $\mu$, and the source vectors of the electric and magnetic current types are denoted $\mathbf{J}^{e}, \mathbf{J}^{m}$. In a horizontally layered model, the medium parameters $\eta=\sigma(z)+i \omega \varepsilon(z)$ and $\zeta=i \omega \mu(z)$ only vary as a function of depth, denoted $z$. Inside any homogeneous subdomain of the layered model, Maxwell's equations can be transformed to the horizontal wavenumber-frequency domain and written as

$$
\begin{gathered}
\eta \tilde{E}_{z}=-\tilde{J}_{z}^{e}-\hat{\mathbf{z}} \cdot\left(i \mathbf{k}_{T} \times \tilde{\mathbf{H}}_{T}\right), \\
\zeta \tilde{H}_{z}=-\tilde{J}_{z}^{m}+\hat{\mathbf{z}} \cdot\left(i \mathbf{k}_{T} \times \tilde{\mathbf{E}}_{T}\right), \\
\eta \tilde{\mathbf{E}}_{T}=-\tilde{\mathbf{J}}_{T}^{e}+\hat{\mathbf{z}} \times \partial_{z} \tilde{\mathbf{H}}_{T}-i \mathbf{k}_{T} \times \hat{\mathbf{z}} \tilde{H}_{z}, \\
\zeta \tilde{\mathbf{H}}_{T}=-\tilde{\mathbf{J}}_{T}^{m}-\hat{\mathbf{z}} \times \partial_{z} \tilde{\mathbf{E}}_{T}+i \mathbf{k}_{T} \times \hat{\mathbf{z}} \tilde{E}_{z},
\end{gathered}
$$

where the unit vector in vertical direction is given by $\hat{\mathbf{z}}=\{0,0,1\}$ and $\mathbf{k}_{T}$ denotes the horizontal wavenumber vector. It can be seen that if in equation A-1 we replace $\tilde{\mathbf{E}}$ by $-\tilde{\mathbf{H}}$ and vice versa, replace $\eta$ by $-\zeta$ and $\tilde{\mathbf{J}}^{e}$ by $\tilde{\mathbf{J}}^{m}$, we obtain equation A-2 and if we make the same substitutions in equation A-3, we obtain equation A-4. This is known as the "electromagnetic equivalence principle" and it implies 
that if we solve an electromagnetic problem for the electric field in terms of electric and magnetic current sources we obtain the solution for the magnetic field by substitution.

Now equation A-4 is multiplied with $i \mathbf{k}_{T}$. to yield

$$
\eta i \mathbf{k}_{T} \cdot \tilde{\mathbf{E}}_{T}=-i \mathbf{k}_{T} \cdot \tilde{\mathbf{J}}_{T}^{e}-\partial_{z} \hat{\mathbf{z}} \cdot\left(i \mathbf{k}_{T} \times \tilde{\mathbf{H}}_{T}\right),
$$

and equation A-4 is multiplied with $i \mathbf{k}_{T} \times$ to result in

$$
\begin{aligned}
\zeta i \mathbf{k}_{T} \times \tilde{\mathbf{H}}_{T} & =-i \mathbf{k}_{T} \times \tilde{\mathbf{J}}_{T}^{m}-i \mathbf{k}_{T} \times \hat{\mathbf{z}} \times \partial_{z} \tilde{\mathbf{E}}_{T} \\
& +i \mathbf{k}_{T} \times i \mathbf{k}_{T} \times \hat{\mathbf{z}} \tilde{E}_{z} .
\end{aligned}
$$

Equation A-6 can be simplified by working out the double vector products and eliminating $\tilde{E}_{z}$ using equation A-1 giving

$$
\begin{aligned}
\zeta i \mathbf{k}_{T} \times \tilde{\mathbf{H}}_{T}= & -i \mathbf{k}_{T} \times \tilde{\mathbf{J}}_{T}^{m}-\partial_{z}\left(i \mathbf{k}_{T} \cdot \tilde{\mathbf{E}}_{T}\right) \hat{\mathbf{z}} \\
& +\frac{\kappa^{2}}{\eta}\left(-\tilde{J}_{z}^{e}-\hat{\mathbf{z}} \cdot\left(i \mathbf{k}_{T} \times \tilde{\mathbf{H}}_{T}\right)\right) \hat{\mathbf{z}}
\end{aligned}
$$

Equation A-7 can be made scalar by multiplying it with $\hat{\mathbf{z}} \cdot$ and we end up with

$$
\begin{aligned}
\zeta \hat{\mathbf{z}} \cdot\left(i \mathbf{k}_{T} \times \tilde{\mathbf{H}}_{T}\right)= & -\hat{\mathbf{z}} \cdot\left(i \mathbf{k}_{T} \times \tilde{\mathbf{J}}_{T}^{m}\right)-\partial_{z}\left(i \mathbf{k}_{T} \cdot \tilde{\mathbf{E}}_{T}\right) \\
& +\frac{\kappa^{2}}{\eta}\left(-\tilde{J}_{z}^{e}-\hat{\mathbf{z}} \cdot\left(i \mathbf{k}_{T} \times \tilde{\mathbf{H}}_{T}\right)\right) .
\end{aligned}
$$

Grouping terms in equations A-5 and A- 8 we find a single set of two coupled first-order equations

$$
\begin{gathered}
\partial_{z}\left(i \mathbf{k}_{T} \cdot \tilde{\mathbf{E}}_{T}\right)+\left(\zeta+\kappa^{2} / \eta\right) \hat{\mathbf{z}} \cdot\left(i \mathbf{k}_{T} \times \tilde{\mathbf{H}}_{T}\right) \\
=-\kappa^{2} \tilde{J}_{z}^{e} / \eta-\hat{\mathbf{z}} \cdot\left(i \mathbf{k}_{T} \times \tilde{\mathbf{J}}_{T}^{m}\right), \\
\partial_{z} \hat{\mathbf{z}} \cdot\left(i \mathbf{k}_{T} \times \tilde{\mathbf{H}}_{T}\right)+\eta\left(i \mathbf{k}_{T} \cdot \tilde{\mathbf{E}}_{T}\right)=-i \mathbf{k}_{T} \cdot \tilde{\mathbf{J}}_{T}^{e} .
\end{gathered}
$$

This is an independent set of two first-order equations that can be solved. Notice that we did not use equation A-2, which means that the vertical magnetic field is not part of this system. Therefore, this is the set of differential equations for the TM-mode. The corresponding set of equations for the TE-mode are obtained using the equivalence principle and are not given here. We can write a general matrix differential equation as

$$
\partial_{z}\left(\begin{array}{l}
u_{1} \\
u_{2}
\end{array}\right)+\left(\begin{array}{cc}
0 & A_{12} \\
A_{21} & 0
\end{array}\right)\left(\begin{array}{l}
u_{1} \\
u_{2}
\end{array}\right)=\left(\begin{array}{l}
s_{1} \\
s_{2}
\end{array}\right) .
$$

For this equation, we have the TM-mode and TE-mode components listed in Table 1. For each mode system of equation to be solved for the whole layered model, boundary conditions are necessary for the fields. It can be seen from equations A-1 and A-2 that in a sourcefree layer $u_{1}=-\eta \tilde{E}_{z}$ for the TM-mode and $u_{1}=\zeta \tilde{H}_{z}$ for the TE-mode, whereas $u_{2}$ is always a linear combination of tangential electric or magnetic field components. All these components are continuous across any horizontal boundary, hence the boundary conditions are that $u_{1}$ and $u_{2}$ are continuous across any source-free boundary. The horizontal electric and magnetic fields are expressed in terms of the mode fields as

$$
\begin{aligned}
\tilde{\mathbf{E}}_{T} & =\frac{i \mathbf{k}_{T} \times \hat{\mathbf{z}} u_{1}^{\mathrm{TE}}-i \mathbf{k}_{T} u_{2}^{\mathrm{TM}}}{\kappa^{2}}, \\
\tilde{\mathbf{H}}_{T} & =\frac{i \mathbf{k}_{T} \times \hat{\mathbf{z}} u_{1}^{\mathrm{TM}}+i \mathbf{k}_{T} u_{2}^{\mathrm{TE}}}{\kappa^{2}} .
\end{aligned}
$$

We may have a last potential problem when evaluating the fields at zero horizontal wavenumber because the mode fields are multiplied with the horizontal wavenumber vector. But this is only an apparent problem because the layered model problem is solved explicitly and the horizontal components of the electric and magnetic fields are obtained from the mode fields eliminating this zero point problem. As an example, we give the solution for an electric current dipole source in a homogeneous medium. For an electric current point source at the horizontal origin, depth level $z^{S}$, and with arbitrary direction $j^{e}$, we can write the electric current source vector in the horizontal wavenumber-frequency domain as $\tilde{\mathbf{J}}^{e}=j^{e} \delta\left(z-z^{S}\right)$. Then the electric field vector can be expressed as the superposition of TM-mode $\tilde{\mathbf{G}}^{\mathrm{TM} e e}$ and TE-mode $\tilde{\mathbf{G}}^{\text {TEee }}$, Green's matrices corresponding to the electric field generated by an electric dipole as

$$
\begin{aligned}
\tilde{\mathbf{E}}\left(\mathbf{k}_{T}, z, z^{s}, \omega\right)= & \tilde{\mathbf{G}}^{\mathrm{TM} e e}\left(\mathbf{k}_{T}, z, z^{s}, \omega\right) \cdot \mathbf{j}^{e} \\
& +\tilde{\mathbf{G}}^{\mathrm{TE} e e}\left(\mathbf{k} T, z, z^{s}, \omega\right) \cdot \dot{j}^{e},
\end{aligned}
$$

where the Green's matrices are given by

$$
\begin{aligned}
\tilde{\mathbf{G}}^{\mathrm{TM} e e}\left(\mathbf{k}_{T}, z, z^{S}, \omega\right) & \left(\begin{array}{ccc}
\frac{\left(i k_{x}\right)^{2} \Gamma^{2}}{\kappa^{2} \eta} & \frac{i k_{x} i k_{y} \Gamma^{2}}{\kappa^{2} \eta} & -\frac{i k_{x} \partial_{z}}{\eta} \\
= & \left(\frac{i k_{x} i k_{y} \Gamma^{2}}{\kappa^{2} \eta} \frac{\left(i k_{y}\right)^{2} \Gamma^{2}}{\kappa^{2} \eta}\right. & -\frac{i k_{y} \partial_{z}}{\eta} \\
-\frac{i k_{x} \partial_{z}}{\eta} & -\frac{i k_{y} \partial_{z}}{\eta} & -\zeta+\eta^{-1} \partial_{z} \partial_{z}
\end{array}\right)
\end{aligned}
$$

and

$$
\begin{aligned}
& \tilde{\mathbf{G}}^{\mathrm{TE} e e}\left(\mathbf{k}_{T}, z, z^{s}, \omega\right) \\
& =\left(\begin{array}{ccc}
\frac{\left(i k_{y}\right)^{2} \zeta}{\kappa^{2}} & -\frac{i k_{x} i k_{y} \zeta}{\kappa^{2}} & 0 \\
-\frac{i k_{x} i k_{y} \zeta}{\kappa^{2}} & \frac{\left(i k_{y}\right)^{2} \zeta}{\kappa^{2}} & 0 \\
0 & 0 & 0
\end{array}\right) \frac{\exp \left(-\Gamma\left|z-z^{S}\right|\right)}{2 \Gamma},
\end{aligned}
$$

with $\Gamma=\sqrt{k_{x}^{2}+k_{y}^{2}+\eta \zeta}$. From these equations, it can be seen that the point $\mathbf{k} T=\mathbf{0}$ presents no problem. The effect of boundary interfaces only introduces reflection and transmission effects and does not change the polynomial behavior at $\mathbf{k}_{T}=\mathbf{0}$. With this analysis, the decomposition of Maxwell's equations in two independent equations that can be solved and are numerically tractable, one for the TM-mode and one for the TE-mode, is complete.

\section{REFERENCES}

Amundsen, L., L. Løseth, R. Mittet, S. Ellingsrud, and B. Ursin, 2006, Decomposition of electromagnetic fields into upgoing and downgoing components: Geophysics, 71, no. 5, G211-G223, doi: 10.1190/1 .2245468 . 
Andréis, D., and L. MacGregor, 2008, Controlled-source electromagnetic sounding in shallow water: Principles and applications: Geophysics, 73, no. 1, F21-F32, doi: 10.1190/1.2815721.

Avdeev, D. B., 2005, Three-dimensional electromagnetic modelling and inversion from theory to application: Surveys in Geophysics, 26, 767799, doi: 10.1007/s10712-005-1836-x.

Berkhout, A. J., 1982, Seismic migration: Imaging of acoustic energy by wave field extrapolation: Elsevier.

Chave, A. D., and C. S. Cox, 1982, Controlled electromagnetic sources for measuring electrical conductivity beneath the oceans: 1 . Forward problem and model study: Journal of Geophysical Research, 87, 5327-5338, doi: 10.1029/JB087iB07p05327.

Constable, S., and L. J. Srnka, 2007, An introduction to marine controlledsource electromagnetic methods for hydrocarbon exploration: Geophysics, 72, no. 2, WA3-WA12, doi: 10.1190/1.2432483.

Eidesmo, T., S. Ellingsrud, L. M. MacGregor, S. Constable, M. C. Sinha, S. Johansen, F. N. Kong, and H. Westerdahl, 2002, Sea bed logging (SBL), a new method for remote and direct identification of hydrocarbon filled layers in deepwater areas: First Break, 20, 144-152.

Fan, Y., R. Snieder, E. Slob, J. Hunziker, and J. Singer, 2011, Steering and focusing diffusive fields using synthetic aperture: Europhysics Letters, 95 34006, doi: 10.1209/0295-5075/95/34006.

Fan, Y., R. Snieder, E. Slob, J. Hunziker, J. Singer, J. Sheiman, and M. Rosenquist, 2010, Synthetic aperture controlled source electromagnetics: Geophysical Research Letters, 37, L13305, doi: 10.1029/ 2010GL043981.

Harris, P., Z. Du, L. MacGregor, W. Olsen, R. Shu, and R. Cooper, 2009 Joint interpretation of seismic and CSEM data using well log constraints: An example from the Luva Field: First Break, 27, 73-81.

Hunziker, J., E. Slob, Y. Fan, R. Snieder, and K. Wapenaar, 2012a, Synthesized 2D CSEM-interferometry using automatic source line determination: 74th Annual International Conference and Exhibition, EAGE, Extended Abstracts, P268.1-P268.5.

Hunziker, J., E. Slob, Y. Fan, R. Snieder, and K. Wapenaar, 2012b, Twodimensional controlled-source electromagnetic interferometry by multidimensional deconvolution: Spatial sampling aspects: Geophysical Prospecting, 60, 974-994, doi: 10.1111/j.1365-2478.2011.01019.x.

Key, K., 2009, 1D inversion of multicomponent, multifrequency marine CSEM data: Methodology and synthetic studies for resolving thin resistive layers: Geophysics, 74, no. 2, F9-F20, doi: 10.1190/1.3058434.
Kinneging, N. A., V. Budejicky, C. P. A. Wapenaar, and A. J. Berkhout, 1989, Efficient 2D and 3D shot record redatuming: Geophysical Prospecting, 37, 493-530, doi: 10.1111/j.1365-2478.1989.tb02220.x.

Kong, J. A., 1986, Electromagnetic wave theory: John Wiley \& Sons.

Lorentz, H. A., 1896, Het theorema van poynting over de energie in het electromagnetisch veld en een paar algemeene stellingen over de voortplanting van het licht: Versl. Koninklijke Nederlandse Akademie van Wetenschappen Amsterdam, 4, 176-188.

MacGregor, L., and M. Sinha, 2000, Use of marine controlled-source electromagnetic sounding for sub-basalt exploration: Geophysical Prospecting, 48, 1091-1106, doi: 10.1046/j.1365-2478.2000.00227.x.

Mittet, R., 2008, Normalized amplitude ratios for frequency-domain CSEM in very shallow water: First Break, 26, 47-54.

Nordskag, J. I., L. Amundsen, L. Løseth, and E. Holvik, 2009, Elimination of the waterlayer response from multi-component source and receiver marine electromagnetic data: Geophysical Prospecting, 57, 897-918, doi: 10.1111/j.1365-2478.2008.00758.x.

Schuster, G., 2009, Seismic interferometry: Cambridge University Press.

Slob, E., 2009, Interferometry by deconvolution of multicomponent multioffset GPR data: IEEE Transactions on Geoscience and Remote Sensing, 47, 828-838, doi: 10.1109/TGRS.2008.2005250.

van den Berg, P. M., A. Abubakar, and T. M. Habashy, 2008, Removal of sea-surface-related wavefields and source replacement in CSEM data processing: 78th Annual International Meeting, SEG, Expanded Abstracts, $672-676$.

Wapenaar, K., D. Draganov, R. Snieder, X. Campman, and A. Verdel, 2010a, Tutorial on seismic interferometry: Part 1 - basic principles and applications: Geophysics, 75, no. 5, 75A195-75A209, doi: 10.1190/1.3457445.

Wapenaar, K., E. Slob, and R. Snieder, 2008, Seismic and electromagnetic controlled-source interferometry in dissipative media: Geophysical Prospecting, 56, 419-434, doi: 10.1111/j.1365-2478.2007.00686.x.

Wapenaar, K., E. Slob, R. Snieder, and A. Curtis, 2010b, Tutorial on seismic interferometry: Part 2 - underlying theory and new advances: Geophysics, 75, no. 5, 75A211-75A227, doi: 10.1190/1.3463440.

Ward, S. H., and G. W. Hohmann, 1988, Electromagnetic theory for geophysical applications, in M. Nabighian, ed., Electromagnetic methods in applied geophysics, 1: SEG.

Wirianto, M., W. A. Mulder, and E. C. Slob, 2011, Exploiting the airwave for time-lapse reservoir monitoring with CSEM on land: Geophysics, 76 no. 3, A15-A19, doi: 10.1190/1.3560157. 